\title{
Value of Combined Detection of Serum CEA, CA72-4, CA19-9 and TSGF in the Diagnosis of Gastric Cancer
}

\author{
Li-Kui Yin ${ }^{1 *}$, Xue-Qing Sun${ }^{1}$, Dong-Zhen Mou
}

\begin{abstract}
Background: To explore whether combined detection of serum tumor markers (CEA, CA72-4, CA19-9 and TSGF) improve the sensitivity and accuracy in the diagnosis of gastric cancer (GC). Materials and Methods: An automatic chemiluminescence immune analyzer with matched kits were used to determine the levels of serum CEA, CA72-4, CA19-9 and TSGF in 45 patients with gastric cancer (GC group), 40 patients with gastric benign diseases (GBD group) hospitalized in the same period and 30 healthy people undergoing a physical examination. The values of those 4 tumor markers in the diagnosis of gastric cancer was analyzed. Results: The levels of serum CEA, CA72-4, CA19-9 and TSGF of the GC group were higher than those of the GBD group and healthy examined people and the differences were significant $(P<0.001)$. The area under receiver operating characteristic (ROC) curves for single detection of CEA, CA72-4, CA19-9 and TSGF in the diagnosis of GC was 0.833, 0.805, 0.810 and 0.839 , respectively. The optimal cutoff values for these 4 indices were $2.36 \mathrm{ng} / \mathrm{mL}, 3.06 \mathrm{U} / \mathrm{mL}, 5.72 \mathrm{U} /$ $\mathrm{mL}$ and $60.7 \mathrm{U} / \mathrm{mL}$, respectively. With combined detection of tumor markers, the diagnostic power of those 4 indices was best, with an area under the ROC curve of $0.913(95 \% \mathrm{CI} 0.866 \sim 0.985)$, a sensitivity of $88.9 \%$ and a diagnostic accuracy of 90.4\%. Conclusions: Combined detection of serum CEA, CA72-4, CA19-9 and TSGF increases the sensitivity and accuracy in diagnosis of $\mathrm{GC}$, so it can be regarded as the important means for early diagnosis.
\end{abstract}

Keywords: Gastric cancer - tumor marker - carbohydrate antigen 72-4 - carcino-embryonic antigen

Asian Pac J Cancer Prev, 16 (9), 3867-3870

\section{Introduction}

Gastric cancer (GC) is one of commonly-seen malignant tumors, with a high incidence rate, and ranks the second of various types of cancer mortality in China (Li et al., 2014; 2015). There were no obvious symptoms in early gastric cancer, but most patients who have been clinically diagnosed are already in middle or advanced stage, which, as a result, is susceptible to delay the best treatment opportunity ( $\mathrm{Li}$ et al., 2015). The early detection, diagnosis and treatment are key to the improvement of curative rate and survival rate of the patients. Although gastroscope is a reasonable method for diagnosis of gastric cancer, it is not suitable for general investigation for patients with subclinical symptoms. A literature has reported that the detection of serum tumor markers is convenient and quick method of easily to be accepted by patients (Tao et al., 2012). At present, on account of lack of specific method for serological diagnosis of GC, the combined detection of serum tumor markers is the effective method for the diagnosis of GC (Yang et al., 2014). In recent years, the detection of tumor markers content is widely applied in clinical diagnosis, so selection of appropriate markers for combined detection provides us a method of dynamically observing the tumor occurrence and progression, and assessing the clinical efficacy and prognosis of the patients, thus increasing the detectable rate and the differential diagnosis accuracy (Fernandes et al., 2005; Jing et al., 2014). In present research, automatic chemiluminescence immune analyzer with matched kits was used to determine the levels of serum carcino-embryonic antigen (CEA), carbohydrate antigen 72-4 (CA72-4), carbohydrate antigen 19-9 (CA19-9) and tumor specific growth factor (TSGF), with the intention to applicative value of combined detection of these 4 indices in the diagnosis of GC.

\section{Materials and Methods}

\section{General data}

Forty-five patients with gastric cancer admitted in Dong Ying People's Hospital from Sep., 2012 to Feb., 2014 were enrolled in GC group. Of all, there were 28 males and 17 females, aged 42 86 years with the average age of $(65.2 \pm 5.7)$ years. Forty patients with gastric benign diseases hospitalized at the same period were selected as GBD group, 24 males and 16 females, aged 39 85 years with the average of $(63.5 \pm 7.8)$ years. Of all, there were 20 patients with gastritis and 20 with gastric ulcer. Additionally, 30 healthy people having physical

${ }^{1}$ Clinical Laboratory, Dong Ying People's Hospital, Dongying, ${ }^{2}$ Department of Immune, Weifang Medical University, Weifang, Shandong, China*For correspondence: yinlikui1234@163.com 
Table 1. Comparison of Serum CEA, CA72-4, CA19-9 and TSGF among 3 Groups $(\bar{x} \pm s)$

\begin{tabular}{lccccc}
\hline Study objects & $\mathrm{n}$ & $\mathrm{CEA}(\mathrm{ng} / \mathrm{mL})$ & $\mathrm{CA} 72-4(\mathrm{U} / \mathrm{mL})$ & CA19-9(U/mL) & TSGF(U/mL) \\
\hline GC group & 45 & $7.57 \pm 3.32^{* \#}$ & $15.38 \pm 7.56^{* \#}$ & $20.58 \pm 0.75^{* \#}$ & $76.19 \pm 11.84^{* *}$ \\
GBD group & 40 & $2.54 \pm 0.34$ & $3.16 \pm 2.02$ & $6.85 \pm 0.98$ & $62.27 \pm 11.45$ \\
Healthy people & 30 & $1.86 \pm 0.22$ & $2.57 \pm 0.78$ & $5.82 \pm 0.46$ & $56.94 \pm 10.66$ \\
\hline
\end{tabular}

${ }^{*}$ GC group refers to patients with GC; GBD group refers to patients with gastric benign diseases. ${ }^{*} P<0.001$ compared with healthy people. ${ }^{*} P<0.001$ compared with GBD group

Table 2. Area Under ROC Curves for Serum CEA, CA72-4, CA19-9 and TSGF

\begin{tabular}{lccccc}
\hline Markers & Area & Std. error & $P$ & \multicolumn{2}{c}{$95 \%$ CI } \\
\cline { 3 - 6 } & & & & $\begin{array}{l}\text { Lower } \\
\text { bound }\end{array}$ & $\begin{array}{r}\text { Upper } \\
\text { bound }\end{array}$ \\
\hline CEA & 0.833 & 0.034 & 0.000 & 0.766 & 0.900 \\
CA72-4 & 0.805 & 0.036 & 0.000 & 0.735 & 0.876 \\
CA19-9 & 0.810 & 0.036 & 0.000 & 0.740 & 0.879 \\
TSGF & 0.839 & 0.033 & 0.000 & 0.775 & 0.903 \\
\hline
\end{tabular}

Table 3. Diagnostic Effectiveness of Single Detection of Serum CEA, CA72-4, CA19-9 and TSGF for GC (\%)

\begin{tabular}{lrlcc}
\hline Markers & $\begin{array}{c}\text { Cut-off } \\
\text { value }\end{array}$ & Sensitivity & Specificity & $\begin{array}{c}\text { Diagnostic } \\
\text { accuracy }\end{array}$ \\
\hline CEA & 2.36 & $66.67(30 / 45)$ & $82.86(58 / 70)$ & $76.52(88 / 115)$ \\
CA72-4 & 3.06 & $64.44(29 / 45)$ & $80.00(56 / 70)$ & $73.91(85 / 115)$ \\
CA19-9 & 5.72 & $68.89(31 / 45)$ & $81.43(57 / 70)$ & $76.52(88 / 115)$ \\
TSGF & 60.67 & $80.00(36 / 45)$ & $82.86(58 / 70)$ & $81.74(94 / 115)$ \\
\hline
\end{tabular}

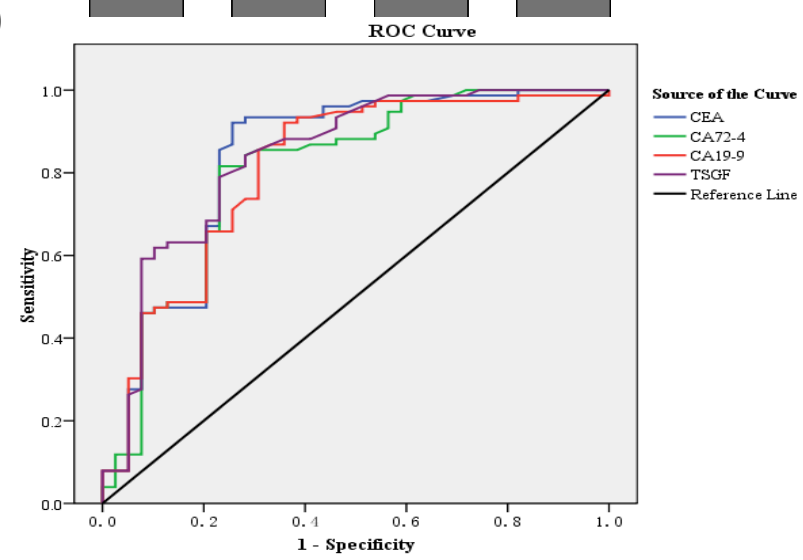

Figure 1. ROC Curves of Single Detection of Serum CEA, CA72-4, CA19-9 and TSGF in Diagnosis of GC. From analysis the curve diagram, the area of 4 indices was over than 0.5 (above the reference line), showing the single detection of them had significance to the differential diagnosis of GC. Of all, the diagnostic power of serum TSGF for GC was the highest one, followed by CEA, CA19-9 and CA72-4

examination at the same period were selected as control, among whom, there were 20 males and 10 females, aged 45 84 years with the average age of $(65.4 \pm 7.2)$ years. There was no difference in baseline data such as age, gender, etc. in three groups $(P>0.05)$, so they were comparable. The present study was approved by ethics committee of Dong Ying People's Hospital. All study objects agreed to participate in the study and signed the informed consent form.

\section{Detection of serum tumor markers}

Fasting elbow venous blood $(4 \mathrm{~mL})$ in the morning was drawn for natural aggregation for $30 \mathrm{~min}$ and centrifuged at $2500 \mathrm{r} / \mathrm{min}$ for $15 \mathrm{~min}$. Separated serum was stored at $-20^{\circ} \mathrm{C}$ until assayed. Automatic chemiluminescence immune analyzer with matched kits was used to determine the levels of serum CEA, CA72-4, CA19-9 and TSGF strictly according to kit introduction. The recommended upper cutoff values for CEA, CA72-4, CA19-9 and TSGF were $3.4 \mathrm{ng} / \mathrm{mL}, 6.9 \mathrm{U} / \mathrm{mL}, 39 \mathrm{U} / \mathrm{mL}$ and $64 \mathrm{U} / \mathrm{mL}$. Testing values over the cutoff value were regarded as positive.

\section{Statistical data analysis}

SPSS 17.0 software package was used for data analysis. The measurement data were presented as mean \pm standard deviation and the comparison among groups was analyzed by $t$ test. receiver operating characteristic (ROC) curves were plotted to determine diagnostic power of CEA, CA72-4, CA19-9 and TSGF and the area under ROC curves and to calculate the optimal cutoff values of single detection and combined detection of these four tumor markers in diagnosis of GC and the sensitivity and specificity at the optimal cutoff values. A values of $P<0.05$ was considered statistically significant.

\section{Results}

Comparison of serum CEA, CA72-4, CA19-9 and TSGF among 3 groups

The levels of serum CEA, CA72-4, CA19-9 and TSGF of GC group were higher than those of GBD group and healthy examined people and the differences were significant $(P<0.001)$. (Table 1$)$.

ROC curves analysis of single detection of serum CEA, CA72-4, CA19-9 and TSGF in the diagnosis of GC

ROC curves analysis showed that the area under ROC curves for serum CEA, CA72-4, CA19-9 and TSGF was $0.833,0.805,0.810$ and 0.839 , respectively. The area of these 4 indices was more than 0.5 , showing that the single detection of them had significance to the differential diagnosis of GC, as shown in Table 2 and Figure 1. According to the results of ROC curves analysis to calculate the optimal cutoff values, the optimal cutoff values for these 4 indices were $2.36 \mathrm{ng} / \mathrm{mL}, 3.06 \mathrm{U} /$ $\mathrm{mL}, 5.72 \mathrm{U} / \mathrm{mL}$ and $60.67 \mathrm{U} / \mathrm{mL}$, respectively, and the corresponding sensitivity, specificity and diagnostic accuracy of the single detection of serum CEA, CA72-4, CA19-9 and TSGF under the optimal cutoff values were as shown in Table 3.

Diagnostic effectiveness of combined detection of serum CEA, CA72-4, CA19-9 and TSGF for GC

Of all types of combined detection of tumor markers, the diagnostic power of combined detection of those 4 indices was best, with the area under ROC curve of 
Table 4. Diagnostic Effectiveness of Combined Detection ofSerum CEA, CA72-4, CA19-9 and TSGF for GC (\%)

\begin{tabular}{lccc}
\hline Markers & Sensitivity & Specificity & Diagnostic accuracy \\
\hline CEA+CA72-4 & $71.11(32 / 45)$ & $88.57(62 / 70)$ & $81.74(94 / 115)$ \\
CEA+ CA19-9 & $75.56(34 / 45)$ & $87.14(61 / 70)$ & $82.61(95 / 115)$ \\
CEA+ TSGF & $80.00(36 / 45)$ & $91.43(64 / 70)$ & $86.96(100 / 115)$ \\
CA72-4+ CA19-9 & $66.67(30 / 45)$ & $85.71(60 / 70)$ & $78.26(90 / 115)$ \\
CA72-4+ TSGF & $71.11(32 / 45)$ & $87.14(61 / 70)$ & $80.87(93 / 115)$ \\
CA19-9+ TSGF & $73.33(33 / 45)$ & $87.14(61 / 70)$ & $81.74(94 / 115)$ \\
CEA+CA72-4+ CA19-9 & $75.56(34 / 45)$ & $90.00(63 / 70)$ & $84.35(97 / 115)$ \\
CEA+CA72-4+ TSGF & $84.44(38 / 45)$ & $91.43(64 / 70)$ & $88.70(102 / 115)$ \\
CA72-4+ CA19-9+ TSGF & $77.78(35 / 45)$ & $92.86(65 / 70)$ & $86.96(100 / 115)$ \\
CEA+CA72-4+ CA19-9+ TSGF & $88.89(40 / 45)$ & $91.43(64 / 70)$ & $90.43(104 / 115)$ \\
\hline
\end{tabular}

0.913 (95\%CI $0.866 \sim 0.985$ ), the sensitivity of $88.89 \%$ and diagnostic accuracy of $90.43 \%$, showing combined detection of 4 indices could improve the differential diagnosis power for GC. (Table 4).

\section{Discussion}

$\mathrm{GC}$ is one of malignant tumors threatening human health. It ranked the third of cancer-related death in Korea in 2010 (Jung et al., 2012; Xu et al., 2014). It was reported that 21320 patients had gastric cancer, with poor prognosis in advanced stage, even lower than 30\% (Siegel et al., 2013; Ji et al., 2014; Liu et al., 2014). Although application of various methods of imaging and endoscopic examinations play important roles in the diagnosis of tumors and make the survival time of GC patients prolonged (Pan et al., 2013; Chen et al., 2014), their examination process is really complex and expensive, especially difficult to realize the early detection, so there is limitation to the early diagnosis, judgment of reoccurrence and evaluation of efficacy for tumors (Park et al., 2014). The detection of tumor markers could reflect the occurrence and development of tumors timely and the detection process is convenient and rapid, with high sensitivity. Hence, they have been the important clinical means of auxiliary examination in the clinical diagnosis and prognosis evaluation for tumors (Femandes et al., 2005; Duraes et al., 2014).

CEA is cancer embryo antigen located on chromosome 19 , commonly used for the diagnosis of malignant tumors of digestive tract, but it isn't a specific index for the diagnosis of other malignant tumors (Gwak et al., 2014). CA19-9 mainly consists of sialic acid and salivary glycoproteins, and significantly increased in the serum in patients with multiple epithelial malignant tumors derived from endodermal cells. CA19-9, closely related to tumor size, lymphatic metastasis and depth of invasion, is an index of judgment of whether direct invasion into adjacent organs exists and evaluation of the prognosis of the patients with GC (Choi et al., 2013; Han et al., 2014). CA72-4 is a kind of high molecular weight glycoprotein antigen. Although it was absent in innocent tumor tissues, effusion and normal tissues, it is differentially specific to gastroenteric tumor, ovarian cancer, breast cancer and liver cancer, especially highly specific to gastric cancer, so it is promisingly used in digestive system neoplasm (Reiter et al., 2000; Mattar et al., 2002). TSGF is vascular endothelial growth factor by a large number of proliferations of malignant cells and peripheral blood capillary. It is a generic term of multiple carbohydrate and its metabolite associated with tumor growth. It is a sort of broad-spectrum serum tumor marker specific to malignant tumors (Robertson et al., 2007).

At present, due to lack of specific method for serological diagnosis of GC, the combined detection of serum tumor markers become the effective method for the diagnosis of GC (Yang et al., 2014). In recent years, the detection of tumor markers content is extensively used in clinical diagnosis, so selection of appropriate markers for combined detection provides us a method of dynamically observing the tumor occurrence and progression, and assessing the clinical efficacy and prognosis of the patients, thus increasing the detectable rate and the differential diagnosis accuracy (He et al., 2013; Yun et al., 2014). Lai et al. (2014) reported that the sensitivity of single detection of lysyl oxidase, CEA, CA724, CA19-9 and CA 125 for lymphatic metastasis in GC was $44.12 \%$, $12.75 \%, 21.57 \%, 23.53 \%$ and $15.69 \%$ and the sensitivity of combined detection of them was up to $79.41 \%$, and that of single detection for peritoneal metastasis in GC was $56.52 \%, 23.91 \%, 34.78 \%, 36.96$ and $34.78 \%$, and the sensitivity of combined detection of them was $91.3 \%$, showing the combined detection of these biomarkers could increase the sensitivity of lymphatic metastasis and peritoneal metastasis in GC.

The present study explored the applicative value of combined detection of 4 tumor markers in the diagnosis of GC through detecting the levels of serum CEA, CA199, CA72-4 and TSGF in patients with GC, gastric benign diseases and healthy people. the results were found that the levels of serum CEA, CA72-4, CA19-9 and TSGF of GC patients were higher than those of patients with gastric benign diseases and healthy examined people and the differences were significant $(P<0.001)$. The area under receiver operating characteristic (ROC) curve for single detection of serum CEA, CA72-4, CA19-9 and TSGF in the diagnosis of GC was $0.833,0.805,0.810$ and 0.839 , respectively. The optimal cutoff values for these 4 indices were $2.36 \mathrm{ng} / \mathrm{mL}, 3.06 \mathrm{U} / \mathrm{mL}, 5.72 \mathrm{U} / \mathrm{mL}$ and $60.67 \mathrm{U} / \mathrm{Ml}$. The area of these 4 indices was more than 0.5 , showing that the single detection of them had significance to the differential diagnosis of GC. Moreover, the sensitivity, specificity and accuracy for the diagnosis of GC were improved under these optimal cutoff values. Of all combined detection of tumor markers, the diagnostic power of combined detection of those 4 indices was 


\section{Li-Kui Yin et al}

best, with the area under ROC curve of 0.913 (95\% CI $0.866 \sim 0.985)$, the sensitivity of $88.89 \%$ and diagnostic accuracy of $90.43 \%$, showing combined detection of 4 indices could improve the sensitivity and the differential diagnosis power for GC.

In conclusion, the detection of serum CEA, CA72-4, CA19-9 and TSGF has significance to the differential diagnosis of GC, and the diagnostic power of serum TSGF for GC was the highest one. Combined detection of these 4 indices together is more effective than single detection and increases the sensitivity and accuracy in diagnosis of GC significantly, so it can be regarded as the important means for the early diagnosis of GC.

\section{References}

Choi AR, Park JC, Kim J, et al (2013). High level of preoperative carbohydrate antigen 19-9 is a poor survival predictor in gastric cancer. World J Gastroenterol, 19, 5302-8.

Chen XJ, Li N, Huang YD, et al (2014). Factors for postoperative gallstone occurrence in patients with gastric cancer: a metaanalysis. Asian Pac J Cancer Prev, 15, 877-81.

Durães C1, Almeida GM, Seruca R, et al (2014). Biomarkers for gastric cancer: prognostic, predictive or targets of therapy? Virchows Arch, 464, 367-78.

Femandes LC, Kin SB, Matos D (2005). Cytokeratins and carcinombryonic antigen in diagnosis staging and prognosis of colorectal adenocarconoma. World J Gastroenterol, 11, 548-55.

Fernandes LC, Kin SB, Matos D (2005). Cytokeratins and carcinoembrayonic antigen in diagnosis, staging and prognosis of colorectal adenocarcinoma. World $J$ Gastroenterol, 11, 548-55.

Gwak HK1, Lee JH, Park SG (2014). Preliminary evaluation of clinical utility of CYFRA 21-1, CA 72-4, NSE, CA19-9 and CEA in stomach cancer. Asian Pac J Cancer Prev, 15, 4933-8.

He CZ, Zhang KH, Li Q, et al (2013). Combined use of AFP, CEA, CA125 and CA19-9 improves the sensitivity for the diagnosis of gastric cancer. BMC Gastroenterol, 13, 87 .

Han ES, Lee HH, Lee JS, et al (2014). At which stage of gastric cancer progression do levels of carcinoembryonic antigen and carbohydrate antigen 19-9 increase? Application in advanced gastric cancer treatment. J Gastric Cancer, 14, 123-8.

Jing JX, Wang Y, Xu XQ, et al (2014). Tumor markers for diagnosis, monitoring of recurrence and prognosis in patients with upper gastrointestinal tract cancer. Asian Pac J Cancer Prev, 15, 10267-72.

Ji AJ, Liu SL, Ju WZ, et al (2014). Anti-proliferation effects and molecular mechanisms of action of tetramethypyrazine on human SGC-7901 gastric carcinoma cells. Asian Pac J Cancer Prev, 15, 3581-6.

Jung KW, Won YJ, Kong HJ, et al (2013). Cancer statistics in Korea: incidence, mortality, survival and prevalence in 2010. Cancer Res and Treat, 45, 1-14.

Li Y, Yang Y, Lu Y, et al (2014). Predictive value of CHFR and MLH1 methylation in human gastric cancer. Gastric Cancer, [Epub ahead of print].

Lai H1, Jin Q, Lin Y, et al (2014). Combined use of lysyl oxidase, carcino-embryonic antigen, and carbohydrate antigens improves the sensitivity of biomarkers in predicting lymph node metastasis and peritoneal metastasis in gastric cancer. Tumour Biol, 35, 10547-54.

Li XF, Liu AY, Li J (2015). Expression of MGMT, hMLH1 and XRCC1 in gastric cancer tissue and their clinical significance. J Int Transl Med, 3, 535-40.

Liu J, Huang XE, Feng JF (2014). Further study on pemetrexed based chemotherapy in treating patients with advanced gastric cancer (AGC). Asian Pac J Cancer Prev, 15, 6587-90.

Mattar R, Alves de Andrade CR, DiFavero GM, et al (2002). Preoperative serum levels of CA72-4, CEA, CA19-9 and alpha-fetoprotein in patients with gastric cancer. Rev Hosp Clin Fac Med Sao Paulo, 57, 89-92.

Pan Z, Pang L, Ding B, et al (2013). Gastric cancer staging with dual energy spectral CT imaging. PLoS One, 8, 53651.

Park K, Jang G, Baek S, et al (2014). Usefulness of combined $\mathrm{PET} / \mathrm{CT}$ to assess regional lymph node involvement in gastric cancer. Tumori, 100, 201-6.

Reiter W, Stieber P, Reuter C, et al (2000). Multivariate analysis of the prognostic value of CEA and CA 19-9 serum levels in colorectal cancer. Anticancer Res, 20, 5195-8.

Robertson DM, Pruysers E (2007). Jobling T. Inhibin in diagnosis for ovarian cancer. Cancer Len, 249, 14-7.

Siegel R, Naishadham D, Jemal A (2013). Cancer statistics, 2013. CA: A Cancer J Clin, 63, 11-30.

Tao XJ, Feng XH, Sun YF, et al (2012). Application of joint detection of CA 72-4, CA19-9, CA50 and CA242 in diagnosis of gastric cancer. Exp and Lab Med, 30, 169-71.

Tian SB, Yu JC, Kang WM, et al (2014). Combined detection of CEA, CA 19-9, CA 242 and CA 50 in the diagnosis and prognosis of resectable gastric cancer. Asian Pac J Cancer Prev. 15, 6295-300.

Whiting J, Sano T, Saka M, et al (2006). Follow-up of gastric cancer: a review. Gastric Cancer, 9, 74-81.

Xu GH, Li Y, Li Y (2014). Expressions of SEL 1L and C-erbB-2 in Gastric Cancer Tissues and Its Clinical Significance. J Int Transl Med, 2, 318-21.

Yang AP, Liu J, Lei HY, et al (2014). CA72-4 combined with CEA, CA125 and CA19-9 improves the sensitivity for the early diagnosis of gastric cancer. Clin Chim Acta, 437, 183-6.

Yun L, Bin Z, Guangqi G, et al (2014). Clinical significance in combined detection of serum pepsinogen I, pepsinogen II and carbohydrate antigen 242 in gastric cancer. Hepatogastroenterology, 61, 255-8. 